\title{
MammoSite Brachytherapy
}

National Cancer Institute

\section{Source}

National Cancer Institute. MammoSite Brachytherapy. NCI Thesaurus. Code C157915.

A method for brachytherapy that uses an implanted catheter to deliver a radioactive seed into the breast site. During a lumpectomy procedure a catheter with a balloon tip is temporarily implanted into the site where the tumor was removed. After surgery a radioactive seed is fed through the catheter to the tumor site. 